Schweiz. Z. Path. Bakt. 1941;4:I-IV

\title{
Contents, Vol. 4, 1941
}

Schweizerische Zeitschrift

für

Pathologic und Bakteriologie

Revue Suisse de Pathologic et de Bactériologie

Unter Mitarbeit von: C. Hallauer P. Hauduroy H. v. Meyenburg J.L. Nicod Th. Reh

Bern Lausanne Zurich Lausanne Geneve

E. Rutishauser C. Wegelin A. Werthemann

Geneve Bern Basel

Herausgegeben von:

A. v. Albertini - A. Grumbach - H. Mooser Zurich

Vol. IV

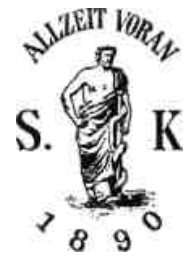

1941

BASEL (Schweiz)

S. K A R G E R

NEW YORK

Holbeiøstraßße $22 \quad 215$ Fourth Avenue

For USA.: Nordeman Publishing, Inc., Dept. S. Karger, 215 Forth Avenue, New York, N. Y.

Alle Rechte vorbehalten

Printed in Switzerland.

Clidiés: Aberegg-Steiner \& Cie. AC, Bern.

Druck $\tau$ on Friedrich Reinhardt in Basel.

INDEX

Vorwort der Herausgeber - Avant-propos des éditeurs . V

Albertini, A. v., Zur FГage der traumatischen Genese der

Pachymeningitis haemorrhagica interna . ․ 442 Barbey, H., Ostéopathie aviaire par carence.

Ses formes et

ses modalités de guérison

121

Bloch, H., Zur Frage der Organspezifität von Nieren-

extrakten 332

Bordet, J. et P., Le role du calcium dans la dissociation

microbienne

321 
Fust, B., Ueber die wachstumshemmende und keimtötende

Wirkung der Dämpfe von Allium sativum (Knoblauch) 340 Grilícheß, R. K., vide Mooser, H. Grumbach, A., vide Ruppaner, E.

- $\quad$ Technik des In-vitro-Versuches zur Auswertung von

Sulfanilamiden 455

Hallauer, C, und Regamey, R., Ueber einen modifizierten

Typhus-Paratyphus-Tetanus- (T.P.T.-) Impfstoff . . 350

Hultquist, G., und Torgersen, O., Zur Kenntnis der Schül-

ler-Christianschen Krankheit beim Erwachsenen . . 193

Kohlschütter, E., and Koppisch, E., On the Mode of Ex

trusion of Schistosome Ova from Blood Vessels into

the Tissues 357

Koppisch, E., vide Kohlschütter, E.

Kuklianskis, J. L., Wirken carcinogene Kohlenwasserstoffe und Sexualhormone karyoklastisch? 167

Lewis, J. H., The Properties of Brain Antigens and their

Antisera 370

Mentha, C., Ostéodystrophies pancréatiques humaines et expérimentales 209

Mooser, H., Experimente zur Frage der epidemiologischen

Bedeutung inapparenter Infektionen beim Fleckfieber 1

- $\quad$ und Grilicheß, R. K., Immunisierungsversuche mit

Steroiden $\quad 375$

Mooser, H., und Leemann, A., Versuche über Immunisierung gegen klassisches und murines Fleckfieber mit toten Impfstoffen 411

Mutsaars, W., et Robert, J., De Гeffet défavorable de Гantisérum spécifique su $\gamma$ la sensibilisation active du cobaye 381 Regamey, R., Studien über die Wirksanıkeit de $\Gamma$ antitetanischen «injection de rappel» bei T.P.T.-Geimpften . . 177

Regamey, R., vide Hallauer, C.

Robert, J., vide Mutsaars, W.

Ruppaner, E., und Grumbach, A., Uebe $\Gamma$ einen außergewöhnlichen bakteriologischen Befund («vibrio septicus Kolle»?) bei einer Gasphlegmone nach Embolektomie $\quad 390$

Scheídegger, S., Amaurotische Idiotie. Ihre Stellung zu den Lipoidosen und die Beziehung zu deГ Niemann-Pick-schen Krankheit, (1-lierzu Tab. I-III) .... 27

Schönberg, S., und Wolf-Heidegger, G., Zur Frage des hypophysären Infantilismus 467

Schwab, R., Uebe $\Gamma$ die Einwirkung von Hormonen auf das

Wachstum des humanen Tuberkelbazillenstammes

Betge 301

Seidenberg, S., Zur Aetiologie deГ Pustulosis vacciniformis acuta 398

Torgersen, 0., vide Hultquist, G. 
Walthard, B. und K., Triorthokresylphosphatvergiftung . 402

Wolf-Heidegger, G., vide Schönberg, S.

NECROLOGIA

Max Askanazy. (1865-1940) F. Griffith $\uparrow \ldots$. . W. Scott $\uparrow \ldots$ 